\title{
A Comparative Study on Current- and Voltage-Optimized Circuit Scheme for Multiple-Transmitter and Single-Receiver WPT System
}

\author{
Jin Zhang $\mathbb{D}^{1,2,3,4}$ Weiao Xu, ${ }^{1}$ Dong Chen $\mathbb{D}^{2,},{ }^{2,5}$ and Chen Zhang ${ }^{2,5}$ \\ ${ }^{1}$ School of Electronic and Information Engineering, Jinling Institute of Technology, Nanjing 211169, China \\ ${ }^{2}$ National and Local Joint Engineering Laboratory of RF Integration and Micro-Assembly Technology, Nanjing 210023, China \\ ${ }^{3}$ School of Electrical Engineering, Southeast University, Nanjing 210096, China \\ ${ }^{4}$ Key Laboratory of Smart Grid Technology and Equipment in Jiangsu Province, Zhenjiang 212000, China \\ ${ }^{5}$ School of Electric Science and Engineering, Nanjing University of Posts and Telecommunications, Nanjing 210023, China \\ Correspondence should be addressed to Jin Zhang; jinzhang@jit.edu.cn
}

Received 30 May 2020; Revised 17 July 2020; Accepted 23 July 2020; Published 13 August 2020

Academic Editor: Hector E. Nistazakis

Copyright (c) 2020 Jin Zhang et al. This is an open access article distributed under the Creative Commons Attribution License, which permits unrestricted use, distribution, and reproduction in any medium, provided the original work is properly cited.

\begin{abstract}
As an important structural system for effectively improving power delivered to the load (PDL) and power transmission range, multiple-transmitter (TX) and single-receiver (RX) wireless power transfer (WPT) system is gaining more and more attention in both academic circles and the industrial fields. Based on the Lagrange multiplier method, this paper first provides a current- and voltage-optimized circuit scheme to maximize the PDL of the multiple-TX WPT system. Then, for a determined WPT system, the current-optimized circuit scheme is proposed to maximize the PDL effectively with constant source voltages and feeding currents for TXs. While voltage-optimized circuit scheme can effectively adjust the source voltages and feeding currents and maintain the same level of input power and PDL as a current-optimized solution. Through comparative study, the voltage-optimized solution shows its advantages in adjustable source voltage and feeding current without any degradation of PDL. Finally, the theoretical analysis results are confirmed by the results of full-wave electromagnetic simulation.
\end{abstract}

\section{Introduction}

In the last ten years, wireless power transfer (WPT) technology based on magnetic coupling has been developed rapidly in both academic and industrial fields. In structure, the classic magnetic resonance-based WPT system was composed of four elements: source loop, transmitter (TX), receiver (RX), and load loop [1-3]. The source and load loop are used for impedance matching to maximize the power delivered to the load (PDL) of the whole system. In order to improve system performance and extend transmission distance, multirepeater WPT systems have been proposed [4-7]. In the multiple-RX scenario, the system of a single charging platform supplying power to multiple devices has been extensively studied by many researchers [8-10]. Some approaches using multiple TXs to transfer more power to a single RX have been studied in [11-14]. In methodology, the early analysis approach of the magnetic coupling WPT system was the coupled-mode theory (CMT) $[1,4,15]$. The circuit theory (CT) has become the primary analysis means in recent years for the parameters with a clear physical meaning. The technologies shaping the magnetic flux in a steerable beam based on CT have been developed in the multiple-TX system [16-18]. The theory of the maximum system power transfer efficiency (PTE) in proportion to the radiation efficiency of the transmitting and receiving antenna has been found and verified in radiating near-field region of the antennas [19-21].

The research work above has greatly contributed to the research of magnetic coupling WPT. The CT analytical method and the multiple-TX and single-RX wireless charging system are applicable to the dynamic charging 
scenario [14, 22, 23]. The current-optimized scheme based on the Lagrange multiplier method in [14] is proposed to tune the currents in multiple TXs to maximize the PDL for the automated guided vehicle (AGV) dynamic charging. Reference [22] focuses on the methods of active charging area determining and output power control to obtain a stable output power for wirelessly dynamic charging EV system. Reference [23] arrived at a conclusion that the maximum PTE of multiple-TX and single-RX WPT system was unaffected by the cross-coupling among TXs. However, to our knowledge, previous research studies focus on optimizing the input currents in TXs to obtain the maximal PDL or PTE. For each optimal current, there is a corresponding optimal source voltage that generates the optimal current. If the optimal current values are higher than the overcurrent of compensating lumped elements, or the equivalent source voltages cannot be quantificationally provided by designed high-frequency inverters, the WPT system becomes corrupted or fails to achieve the transmission power setting. Therefore, an adjustable feeding current and source voltage WPT system needs to be studied to achieve targeted transmission power without electrical element damage and system crash.

In this paper, the maximum PDL is first obtained by optimizing feeding currents by using series capacitor compensation separately at the transmit and the receive ends. Then, the series inductor/shunt capacitor/series capacitor (LCC) compensation topology at the transmit end is proposed to obtain adjustable source voltage and feeding current. The current optimal scheme is converted into voltage one by using the LCC compensation technique.

This paper is organized as follows. The circuit model and optimal approaches of the multiple-TX WPT system are presented in Section 2. In this section, the analysis and comparison of two cases of equal and optimal current (EC and OC Cases) are illustrated to highlight the superiority of the current-optimized methodology. In Section 3, the voltage-optimized circuit scheme of the multiple-TX WPT system is proposed via the two corresponding voltage optimal cases (EV and OV Cases). The analysis and comparison between the two optimization schemes are presented in Section 4. In Section 5, the results of the theoretical calculation are executed by MATLAB, and full-wave electromagnetic simulation is carried out by FEKO software to validate the theoretical calculation. Finally, Section 6 contains some concluding remarks.

\section{Current-Optimized Circuit Scheme of Multiple-TX WPT}

The compensation capacitors are connected to transmitting and receiving inductors in series in Figure 1. The currentoptimized scheme is implemented based on capacitor series topology, which is presented in this section.

According to Kirchhoff's circuit laws, the electrical properties for multi-TX WPT system in Figure 1 can be described by the following formula:

$$
\left[\frac{1}{r_{R}^{\prime}}\left[\begin{array}{cccc}
r_{T 1} & j \omega_{0} M_{T 1 T 2} & \ldots & j \omega_{0} M_{T 1 T n} \\
j \omega_{0} M_{T 1 T 2} & r_{T 2} & \ldots & j \omega_{0} M_{T 2 T n} \\
\vdots & \vdots & \vdots & \ddots \\
j \omega_{0} M_{T 1 T n} & j \omega_{0} M_{T 2 T n} & \cdots & r_{T n}
\end{array}\right]_{-j Q_{T n R}}^{-j Q_{T 1 R}}\right.
$$

where $M_{T i T j}(i, j=1, \ldots, n, i \neq j)$ is the mutual inductance between $\mathrm{TX}_{i}$ and $\mathrm{TX}_{j}, V_{T i}$ and $I_{T i}$ are the RMS values of the source voltage and loop current, respectively, and $r_{T X i}$ is the parasitic resistance of $\mathrm{TX}_{i} ; I_{C, R}$ and $r_{R}^{\prime}$ are the loop current and total resistance of the RX. $r_{R}^{\prime}$ can be written as $r_{R}^{\prime}=r_{R}+r_{L}$, where $r_{R}$ and $r_{L}$ are the parasitic resistance of the RX and the resistance of the load. $Q_{T i R}=\left(\omega_{0} M_{T i R} / r_{R}^{\prime}\right)$ is defined as transmission quality factor, where $\omega_{0}=\left(1 / \sqrt{L_{T i} C_{T i}}\right)$ and $M_{T i R}$ are the angular resonance frequency and the mutual inductance between $\mathrm{TX}_{i}$ and $\mathrm{RX}$, respectively. It can be found that $Q_{T i R}$ indicates the coupling strengths when RX and load are fixed.

2.1. Theoretical Derivation. The first $n$ lines and the last line of equation (1) can be written in equations (2a) and (2b), respectively, as follows:

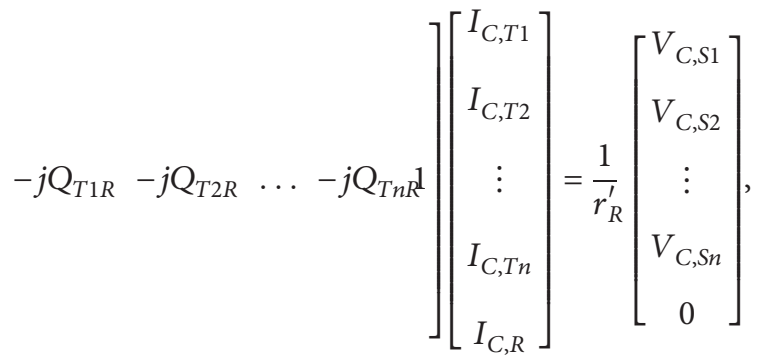

$$
r_{T i} \mathbf{I}_{C, T i}+j \omega_{0} \sum_{j=1, j \neq i}^{n}\left(M_{T i T j} \mathbf{I}_{C, T j}\right)-r_{R}^{\prime} j Q_{T i R} \mathbf{I}_{C, R}=\mathbf{V}_{C, S i},
$$

$$
\mathbf{I}_{C, R}-j \sum_{i=1}^{n}\left(Q_{T i R} \mathbf{I}_{C, T i}\right)=0 .
$$

$\mathrm{PDL}_{C}$ for the current-optimized scheme, $\mathrm{PDL}_{C}=r_{L}\left|\mathbf{I}_{C, R}\right|^{2}$, can be achieved via (2b):

$$
\mathrm{PDL}_{C}=r_{L}\left(\sum_{i=1}^{n} Q_{T i R} I_{C, T i}\right)^{2}
$$

$\mathbf{V}_{C, S i}$ can be expressed explicitly in terms of $\mathbf{I}_{C, T i}$ using equations of (2a) and (2b). The power gained from the $i$-th 


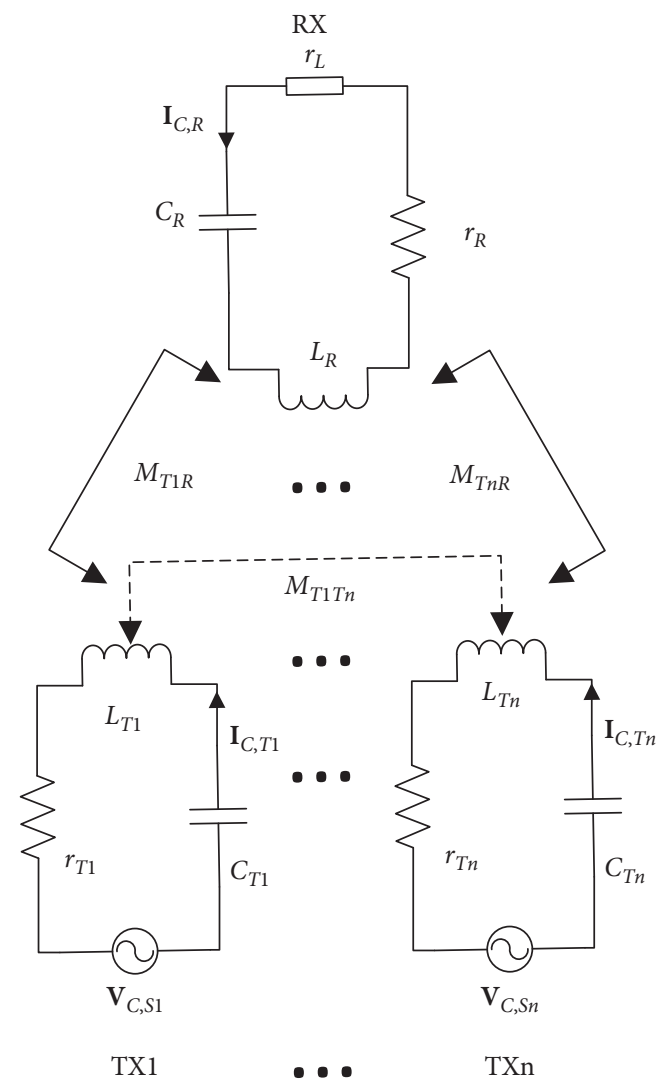

FIGURE 1: Circuit topology of the current-optimized circuit scheme of multiple-TX WPT system.

source, $P_{C, T i}$, for the current-optimized scheme is calculated by $P_{C, T i}=\operatorname{Re}\left(\mathbf{V}_{C, S i} \mathbf{I}_{C, T i}^{*}\right)$.

$P_{C, T i}=\left(r_{T i}+r_{R}^{\prime} Q_{T i R}^{2}\right)\left|\mathbf{I}_{C, T i}\right|^{2}+r_{R}^{\prime} Q_{T i R}\left(\sum_{j=1, j \neq i}^{n} Q_{T j R} \mathbf{I}_{C, T j}\right) \mathbf{I}_{C, T i}$.
The detailed calculation of total feeding power to the WPT network, $P_{C, T}=\sum_{i=1}^{n} P_{C, T i}$, is as follows:

$$
P_{C, T}=\sum_{i=1}^{n} r_{T i} \mathbf{I}_{C, T i}^{2}+r_{R}^{\prime}\left(\sum_{i=1}^{n} Q_{T i R} \mathbf{I}_{C, T i}\right)^{2}
$$

For purposes of comparison, equal current (EC Case) flowing through each TX for the multiple-TX WPT system is presented. The identical current, $\mathbf{I}_{C, T, I}$, can be derived from equation (5):

$$
\mathbf{I}_{C, T, I}=\frac{\sqrt{P_{C, T}}}{\sqrt{\sum_{i=1}^{n} r_{T i}+r_{R}^{\prime}}\left(\sum_{i=1}^{n} Q_{T i R}\right)^{2}} .
$$

Combining equation (3) and (6), the PDL for the conventional multiple-TX system with the identical current in TXs is derived in

$$
\mathrm{PDL}_{C, I}=\frac{P_{C, T} r_{L}}{r_{R}^{\prime}+\left(1 / F_{1}\right)},
$$

where $F_{1}=\left(\left(\sum_{i=1}^{n} Q_{T i R}\right)^{2} / \sum_{i=1}^{n} r_{T i}\right)$.

In order to maximize PDL via optimizing $\mathbf{I}_{C, T i}$ in each TX under the equality constraint of (5), we achieve the optimized objective functions as follows:

$$
\begin{array}{ll}
\underset{\mathbf{I}_{T i}}{\operatorname{Maximize}} & r_{L}\left(\sum_{i=1}^{n} Q_{T i R} \mathbf{I}_{C, T i}\right)^{2} \\
\text { Subject to } & \sum_{i=1}^{n} r_{T i} \mathbf{I}_{\mathrm{C}, \mathrm{Ti}}^{2}+r_{R}^{\prime}\left(\sum_{i=1}^{n} Q_{T i R} \mathbf{I}_{C, T i}\right)^{2}-P_{C, T}=0 .
\end{array}
$$

The optimal solutions of $\mathbf{I}_{C, T i \text {,OPT }}$ for (8a) and (8b) could be obtained using the Lagrange multiplier method. Introducing the variable in the set of real numbers, $\lambda$, the optimization of (8a) and (8b) is constructed by the Lagrange multiplier equation:

$$
L\left(\mathbf{I}_{\mathbf{C}, \mathbf{T i}}, \lambda\right)=r_{L}\left(\sum_{i=1}^{n} Q_{T i R} \mathbf{I}_{\mathbf{C}, \mathbf{T i}}\right)^{2}+\lambda\left[\sum_{i=1}^{n} r_{T i} \mathbf{I}_{\mathbf{C}, \mathbf{T i}}^{2}+r_{R}^{\prime}\left(\sum_{i=1}^{n} Q_{T i R} \mathbf{I}_{\mathbf{C}, \mathbf{T i}}\right)^{2}-P_{C, T}\right]
$$

The necessary conditions of optimal solutions for (9) are $\nabla_{\mathbf{I}_{C, T i}} L\left(\mathbf{I}_{C, T i}, \lambda\right)=0$ and $\nabla_{\lambda} L\left(\mathbf{I}_{C, T i}, \lambda\right)=0$, the corresponding

$$
\begin{gathered}
\mathbf{I}_{C, T i}=\frac{Q_{T i R}\left(r_{L}-r_{R}^{\prime} \lambda\right)}{\lambda r_{T i}}\left(\sum_{j=1}^{n} Q_{T j R} \mathbf{I}_{C, T j}\right), \\
\sum_{i=1}^{n} r_{T i} \mathbf{I}_{C, T i}^{2}+r_{R}^{\prime}\left(\sum_{i=1}^{n} Q_{T i R} \mathbf{I}_{C, T i}\right)^{2}-P_{C, T}=0 .
\end{gathered}
$$
expansions of which are listed as follows: 
From (10a), the relations of the current flowing in different loops are deduced of $\left(\mathbf{I}_{C, T i} / \mathbf{I}_{C, T j}\right)=\left(\left(Q_{T i R} /\right.\right.$ $\left.\left.r_{\mathrm{Ti}}\right) /\left(Q_{\mathrm{TjR}} / r_{\mathrm{Tj}}\right)\right) \forall i \neq j$. Substituting the relations of
$\left(\mathbf{I}_{C, T i} / \mathbf{I}_{C, T j}\right)$ into (10b), the optimal current (OC Case) in the $i$-th TX, $\mathbf{I}_{C, T i, O P T}$, is

$$
\mathbf{I}_{C, T i, \mathrm{OPT}}=\frac{\sqrt{P_{C, T}} Q_{T i R}\left(\prod_{j=1, j \neq i}^{n} r_{T j}\right)}{\sqrt{\left[\sum_{s=1}^{n} Q_{T s R}^{2}\left(\prod_{j=1, j \neq s}^{n} r_{T j}\right)\right]\left\{\prod_{j=1}^{n} r_{T j}+r_{R}^{\prime}\left[\sum_{s=1}^{n} Q_{T s R}^{2}\left(\prod_{j=1, j \neq s}^{n} r_{T j}\right)\right]\right\}}}
$$

By substituting $\mathbf{I}_{C, T i, \text { OPT }}$ of (11) into (3), one can derive the optimal PDL of multi-TX, $\mathrm{PDL}_{\mathrm{C}, \mathrm{OPT}}$.

$$
\mathrm{PDL}_{C, \mathrm{OPT}}=\frac{P_{C, T} r_{L}}{r_{R}^{\prime}+\left(1 / F_{2}\right)}
$$

where $F_{2}=\sum_{i=1}^{n}\left(Q_{T i R}^{2} / r_{T i}\right)$.

In order to compare $\mathrm{PDL}_{I}$ of EC Case in equation (7) and the optimal $\mathrm{PDL}_{\mathrm{OPT}}$ of $\mathrm{OC}$ Case in equation (12) for the multi-TX system, the only difference lies in $F_{1}$ and $F_{2}$. To order to compare the numeric value of $\mathrm{PDL}_{I}$ and $\mathrm{PDL}_{\mathrm{OPT}}$, $\Delta F=\left(F_{2}-F_{1}\right)$ is implemented as follows:

$$
\Delta F=\sum_{i=1, i \neq j}^{n}\left[\frac{\left.\left(Q_{T i R} r_{T j}-Q_{T j R} r_{T i}\right)^{2}\left(\prod_{s=1, s \neq i, \neq j}^{n} r_{T s}\right)\right]}{\left(\sum_{i=1}^{n} r_{T i}\right)\left(\prod_{i=1}^{n} r_{T i}\right) .}\right.
$$

There are many groups of $\left(Q_{T i R} r_{T j} \neq Q_{T j R} r_{T i}\right)$ for different sizes of the loop in the multi-TX system; therefore, $\Delta F>0$ is always tenable. Up to this point, the conclusion that the value of $\mathrm{PDL}_{\mathrm{OPT}}$ of the proposed multi-TX system with a current-controlled technique must be greater than that of $\mathrm{PDL}_{I}$ of conventional multi-TX system with the identical feeding current is true.

According to equations (11) and (12), the capacitor series topology for the multi-TX WPT system can maximize the PDL by optimizing currents in TXs. More generally, the optimal feeding currents are more commonly converted to voltage forms to use the voltage source in a practical feeding system. The feeding voltages of $\mathbf{V}_{C, S i}$ and $\mathbf{V}_{C, S i, \mathrm{OPT}}$ for PDL $\mathrm{P}_{C, I}$ and $\mathrm{PDL}_{C, \mathrm{OPT}}$ can be achieved by substituting $\mathbf{I}_{C, T, I}$ and $\mathbf{I}_{C, T i, \mathrm{OPT}}$ into equations (2a) and (2b), respectively. The $i$ th voltage feeding to $\mathrm{TX}_{i}$ is written as

$$
\begin{gathered}
\mathbf{V}_{C, S i}=r_{T i} \mathbf{I}_{C, T, I}+r_{R X}^{\prime} Q_{T i R} \sum_{j=1}^{n} Q_{T j R} \mathbf{I}_{C, T, I}+j \omega_{0} \sum_{j=1, j \neq i}^{n} M_{T i T j} \mathbf{I}_{C, T, I}, \\
\mathbf{V}_{C, S i, \mathrm{OPT}}=r_{T i} \mathbf{I}_{C, T i, \mathrm{OPT}}+r_{R X}^{\prime} Q_{T i R} \sum_{j=1}^{n} Q_{T j R} \mathbf{I}_{C, T j, \mathrm{OPT}}+j \omega_{0} \sum_{j=1, j \neq i}^{n} M_{T i T j} \mathbf{I}_{C, T j, \mathrm{OPT}} .
\end{gathered}
$$

However, the voltage sources are more commonly used to feeding power in the practical WPT system. We investigate the voltage-optimized scheme in Section 3.

2.2. Numerical Analysis. In order to illustrate the effectiveness of the analysis in Section 2.1, we present a WPT system consisting of a maximum of five identical TXs and a single RX. All the diameters and the turn numbers of TXs and $\mathrm{RX}$ are $31 \mathrm{~cm}$ and 25, respectively. We use a $1.2 \mathrm{~mm}$ diameter copper wire with electrical conductivity of $5.7 \times 10^{7} \mathrm{~S} / \mathrm{m}$ to wind the power transmission coils, the selfinductance and parasitic resistance values of which are $L_{T}=$ $L_{R}=40.55 \mu \mathrm{H}$ and $r_{T}=r_{R}=1.96 \Omega$ according to theoretic calculation $[14,24]$. The series capacitors of $C_{T}=C_{R}=625$ $\mathrm{pF}$ are connected to coils to achieve the resonance frequency $f_{0}=1 \mathrm{MHz}\left(\omega_{0}=6.28 \times 10^{6} \mathrm{rad} / \mathrm{s}\right)$.

Figure 2 illustrates the model setup for a maximum of five TXs and a single-RX WPT system [14], which is also investigated in this paper. The parameters of $D_{I}, D_{M}$, and $D_{T}$ are the interval distance between TXs, the offset distance from $\mathrm{RX}$ to $\mathrm{TX}_{3}$, and the transmission distance between TXs and $\mathrm{RX}$, respectively. The total feeding power is set to $P_{C, T}=20 \mathrm{~W}$ for 1-, 2-, 3-, 4-, and 5-TX system, respectively. $D_{I}=0.5 \mathrm{~m}$ is always true in further examples in the following sections. In this theoretical study, MATLAB optimization toolbox is used to analyze the power transmission performance of the presented optimization methods. Figure 3 presents currents among different transmitting coils for EC Case and OC Case when $r_{L}=100 \Omega$. The current among $\mathrm{TX}_{i}$ is symmetrical about the location where the i-th TX sits, whether for EC Case or for OC Case. The variation magnitudes of $\mathbf{I}_{C, T, I}$ and $\mathbf{I}_{C, T i, \mathrm{OPT}}$ for $D_{T}=0.3 \mathrm{~m}$ case are obviously larger than those for $D_{T}=0.7 \mathrm{~m}$ case.

Figure 4 shows the PDLs for EC Case and OC Case. $\mathrm{PDL}_{C, I}$ achieves the maximum value at the position of $D_{M}=-0.1 \mathrm{~m}$ where $\mathrm{TX}_{1}$ is located. As the number of TXs increases, the maximum value of $\mathrm{PDL}_{C, I}$ for $\mathrm{EC}$ Case gradually decreases; however, the valid changing range became distinctly wider. For OC Case, the maximum values of PDL $\mathrm{P}_{\mathrm{C}, \mathrm{OPT}}$ for 1-, 2-, 3-, 4-, and 5-TX systems are the same. Whether in EC Case or OC Case, the fluctuation ranges of the PDL are smaller than those for $D_{T}=0.3 \mathrm{~m}$ case. However, the valid values of PDL for $D_{T}=0.7 \mathrm{~m}$ case are lower 


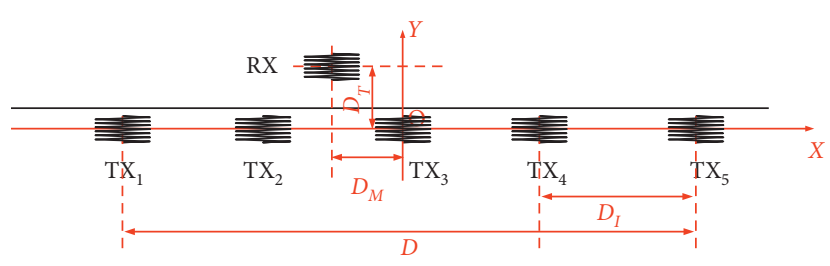

Figure 2: Multiple-TX and single-RX model setup.
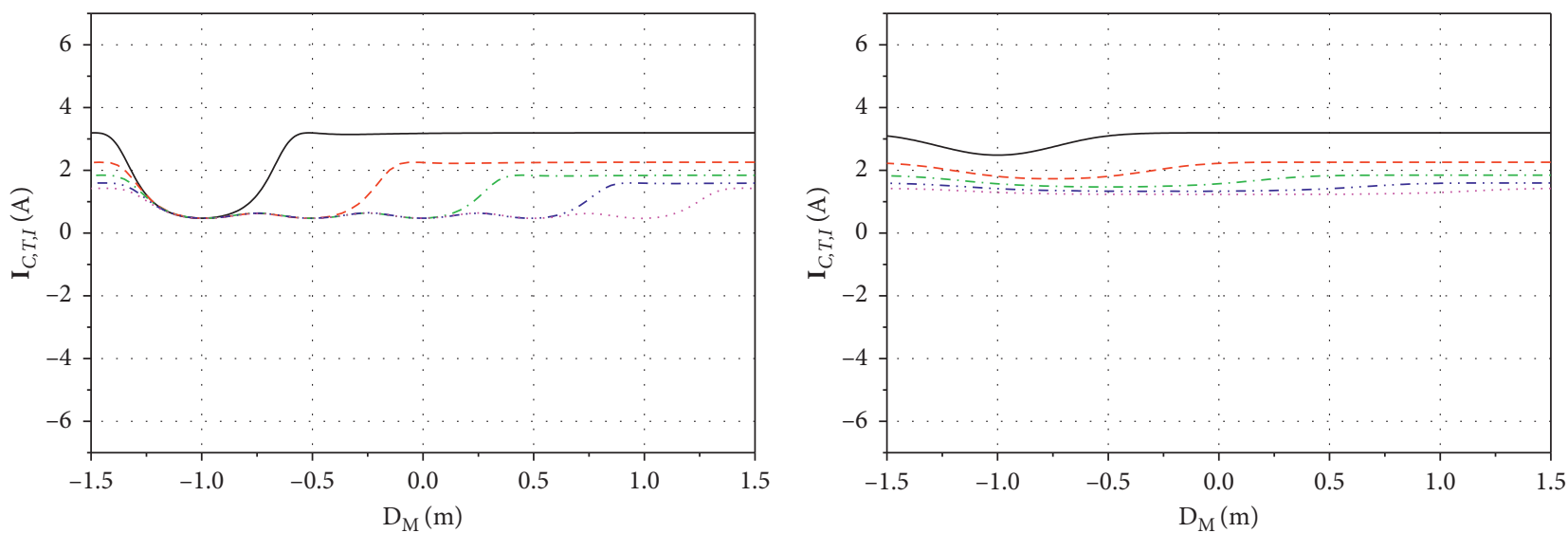

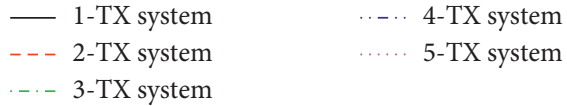

(a)

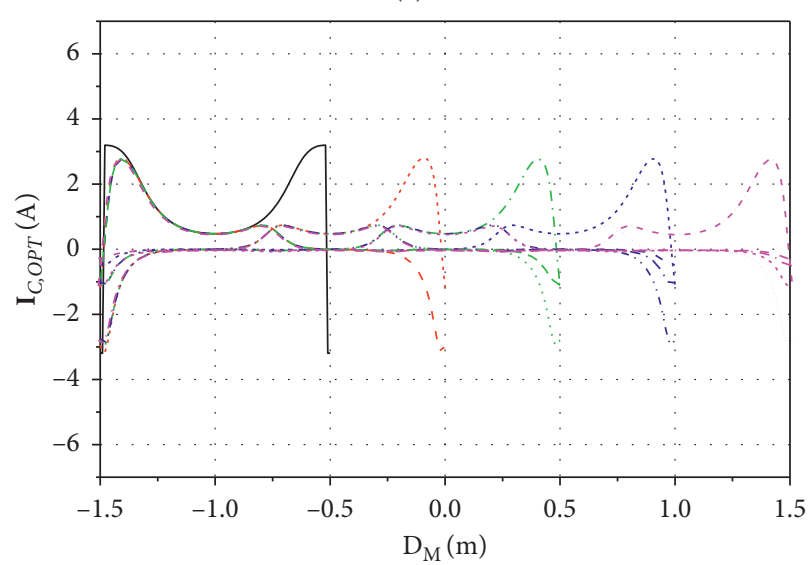

1-TX system

$-\mathbf{I}_{C, T 1, \mathrm{OPT}}$

3-TX system

$\cdot-\cdot \mathbf{I}_{C, T 1, \mathrm{OPT}}$

- - $\mathbf{I}_{C, T 2, \mathrm{OPT}}$

$\mathbf{I}_{C, T 3, \mathrm{OPT}}$

5-TX system

$--\mathbf{I}_{C, T 1, \mathrm{OPT}}$

$\ldots \mathbf{I}_{C, T 2, \mathrm{OPT}}$

$\mathbf{I}_{C, T 3, \mathrm{OPT}}$

$-\mathbf{I}_{C, T 4, \mathrm{OPT}}$

.... $\mathbf{I}_{C, T 5, \mathrm{OPT}}$
2-TX system

- - - $\mathbf{I}_{C, T 1, \mathrm{OPT}}$

$\mathbf{I}_{C, T 2, \mathrm{OPT}}$

4-TX system

- - $\mathbf{I}_{C, T 1, \mathrm{OPT}}$

-.. $\mathbf{I}_{C, T 2, \mathrm{OPT}}$

$\ldots \mathbf{I}_{C, T 3, \mathrm{OPT}}$

$\ldots . . \mathbf{I}_{C, T 4, \mathrm{OPT}}$

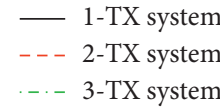

-. - 3-TX system

(b)

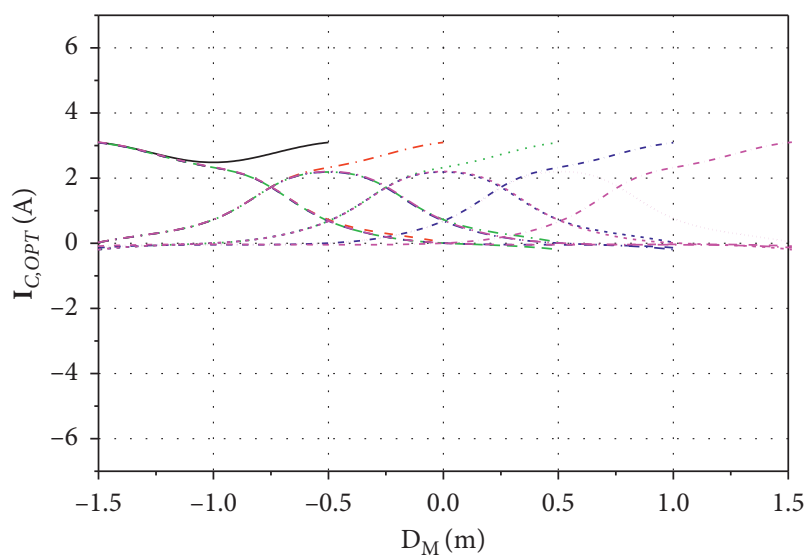

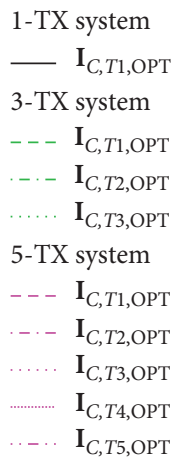

(c)

(d)

Figure 3: The currents among TXs versus $D_{M}$. EC Case when (a) $D_{T}=0.3 \mathrm{~m}$ and (b) $D_{T}=0.7 \mathrm{~m}$. OC Case when (c) $D_{T}=0.3 \mathrm{~m}$ and (d) $D_{T}=0.7 \mathrm{~m}$. 


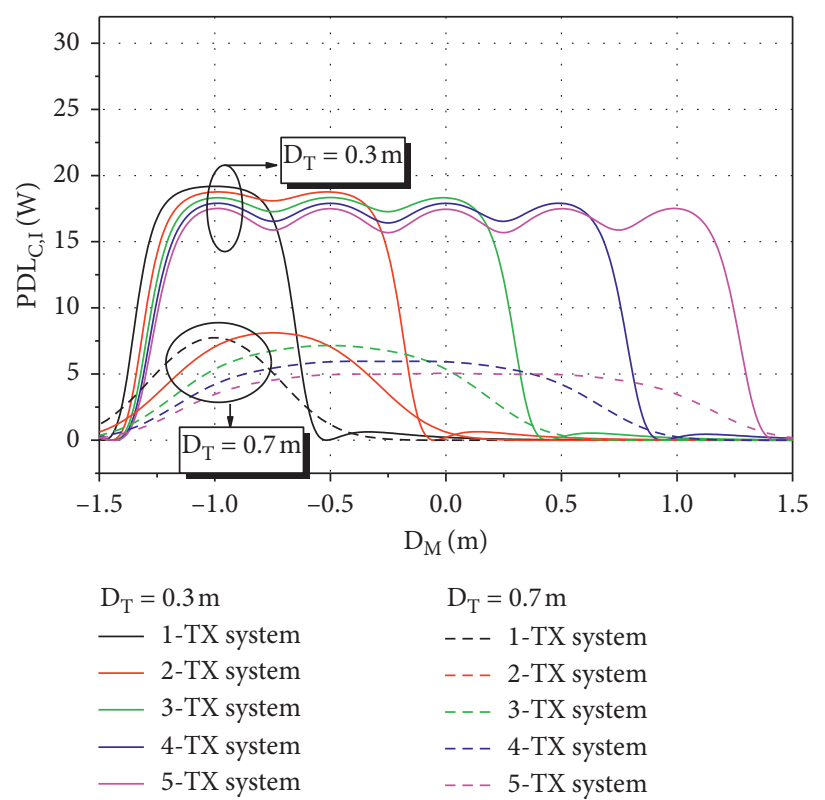

(a)

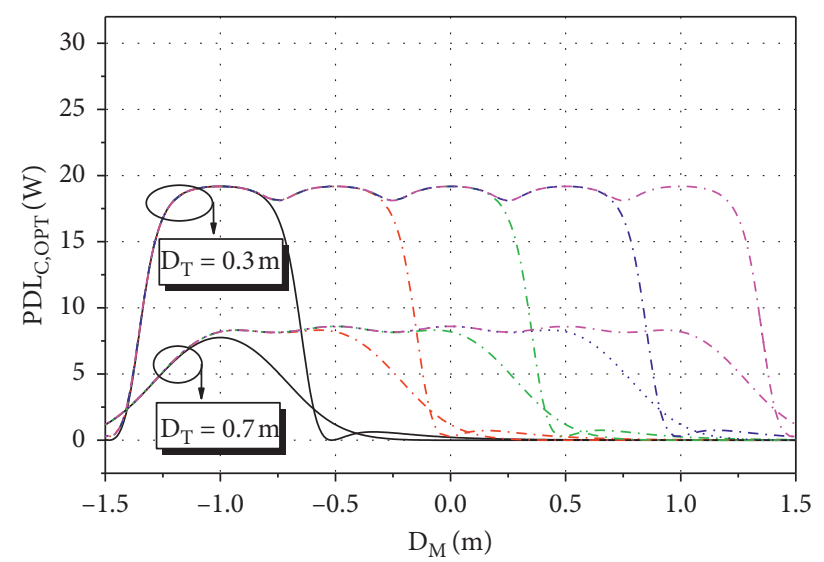

$$
\begin{aligned}
& \mathrm{D}_{\mathrm{T}}=0.3 \mathrm{~m} \quad \mathrm{D}_{\mathrm{T}}=0.7 \mathrm{~m} \\
& \text { - 1-TX system — 1-TX system } \\
& \text { - - 2-TX system - } \quad \text { 2-TX system } \\
& \text {-.- 3-TX system ... 3-TX system } \\
& \text {... 4-TX system …. 4-TX system } \\
& \text {..- 5-TX system ...- 5-TX system }
\end{aligned}
$$

(b)

Figure 4: The PDLs for (a) EC Case and (b) OC Case under the $P_{C, T}=20 \mathrm{~W}$.

than those for $D_{T}=0.3 \mathrm{~m}$ case. By comparing Figure 4(a) with Figure 4(b), the OC Case can obviously improve the PDLs, especially for the system with multiple TXs.

\section{Voltage-Optimized Circuit Scheme of Multiple-TX WPT}

In this section, the series capacitor compensation structures of the transmitting circuit in Section 2 are replaced with inductor/capacitor/capacitor (LCC) compensation networks. Figure 5 shows the LCC compensation topology of the multiple-TX and single-RX WPT system. This system with LCC compensation is used to maximize the PDL based on the voltage-optimized scenario. Under the design equation of (15), the transmitting currents of $I_{V, T i}$ based on LCC compensation are completely determined by feeding voltage values regardless of the load conditions and the coupling strengths between TXs and RX $[25,26]$.

$$
\omega_{0}=\frac{1}{\sqrt{L_{C i} C_{C i}}}=\frac{1}{\sqrt{\left(L_{T i}-L_{C i}\right) C_{T i}}} .
$$

Similarly to the current-optimized circuit scheme in Section 2, the electrical properties between the i-th $\mathrm{TX}_{i}$ and the RX in Figure 2 can be described by Kirchhoff's circuit laws in equation (15).

$$
\begin{gathered}
-\frac{1}{j \omega_{0} C_{C i}} \mathbf{I}_{V, I i}=\mathbf{V}_{V, S i}, \\
r_{T i} \mathbf{I}_{V, T i}-\frac{1}{j \omega_{0} C_{C i}} \mathbf{I}_{V, I i}+j \omega_{0} \sum_{j=1, j \neq i}^{n}\left(M_{T i T j} \mathbf{I}_{V, T j}\right)-r_{R}^{\prime} j Q_{T i R} \mathbf{I}_{V, R}=0 \\
\mathbf{I}_{V, R}-j \sum_{i=1}^{n}\left(Q_{T i R} \mathbf{I}_{V, T i}\right)=0 .
\end{gathered}
$$

$\mathrm{PDL}_{V}=r_{L}\left|\mathbf{I}_{V, R}\right|^{2}$ for the voltage-optimized scheme can be achieved by solving equations 16(a) and 16(c).

$$
\mathrm{PDL}_{V}=\omega_{0}^{2} r_{L}\left(\sum_{i=1}^{n} C_{C i} Q_{T i R} \mathbf{V}_{V, S i}\right)^{2}
$$

The power gained from the $i$-th source, $P_{V, T i}$, is calculated by $P_{V, T i}=\operatorname{Re}\left(\mathbf{V}_{V, S i} \mathbf{I}_{V, I i}^{*}\right)$ using equations (16a), (16b), and (16c):

$$
P_{V, T i}=\omega_{0}^{2}\left(r_{T i}+r_{R}^{\prime} Q_{T i R}^{2}\right) C_{C i}^{2} \mathbf{V}_{V, S i}^{2}+\omega_{0}^{2} r_{R}^{\prime} Q_{T i R}\left(\sum_{j=1, j \neq i}^{n} C_{C j} Q_{T j R} \mathbf{V}_{V, S j}\right) C_{C i} \mathbf{V}_{V, S i}
$$




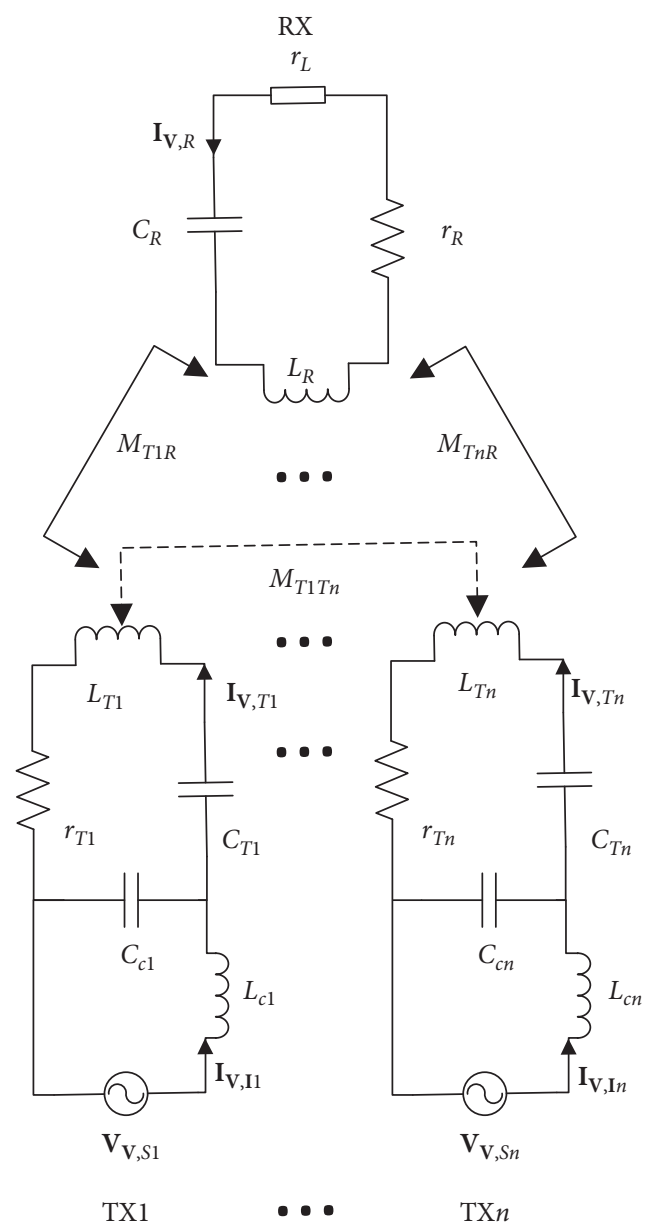

FIGURE 5: Circuit topology of voltage-optimized circuit scheme of multiple-TX WPT system.

The total power supplied by $n$ voltage sources is readily observed by $P_{V, T}=\sum_{i=1}^{n} P_{V, T i}$ :

$$
P_{V, T}=\omega_{0}^{2} \sum_{i=1}^{n} C_{C i}^{2} r_{T i} \mathbf{V}_{V, S i}^{2}+\omega_{0}^{2} r_{R}^{\prime}\left(\sum_{i=1}^{n} C_{C i} Q_{T i R} \mathbf{V}_{V, S i}\right)^{2}
$$

For the equal voltage (EV Case) feeding by each source, the identical voltage, $\mathbf{V}_{V, S, I}$, can be derived from equation (19):

$$
\mathbf{V}_{\mathbf{V}, \mathbf{S}, \mathbf{I}}=\frac{\sqrt{P_{V, T}}}{\omega_{0} \sqrt{\sum_{i=1}^{n} C_{C i}^{2} r_{T i}+r_{R}^{\prime}}\left(\sum_{i=1}^{n} C_{C i} Q_{T i R}\right)^{2}} .
$$

Submitting equation (20) into (17), the PDL for the multiple-TX system with identical feeding voltage is derived in

$$
\mathrm{PDL}_{V, I}=\frac{P_{V, T} r_{L}}{r_{R}^{\prime}+\left(1 / F_{1}\right)}
$$

Similarly to the current-optimized scheme, we maximize equation (17) under the constraint of equation (19) using the Lagrange multiplier method, and the optimal feeding voltages, $\mathbf{V}_{V, S i, O P T}(\mathrm{OV}$ Case) for maximizing PDL, $\mathrm{PDL}_{V, \mathrm{OPT}}$, can be readily observed:

$$
\mathbf{V}_{V, S i, \mathrm{OPT}}=\frac{\sqrt{P_{V, T}} Q_{T i R}\left(\prod_{j=1, j \neq i}^{n} r_{T j}\right)}{\omega_{0} \sqrt{\left[\sum_{s=1}^{n} C_{C s} Q_{T s R}^{2}\left(\prod_{j=1, j \neq s}^{n} r_{T j}\right)\right]\left\{\sum_{s=1}^{n} C_{C s}\left(\prod_{j=1}^{n} r_{T j}\right)+r_{R}^{\prime}\left[\sum_{s=1}^{n} C_{C s} Q_{T s R}^{2}\left(\prod_{j=1, j \neq s}^{n} r_{T j}\right)\right]\right\}}}
$$


Submitting equation (22) into (17), the $\mathrm{PDL}_{V \text {,OPT }}$ for the multiple-TX system with optimal feeding voltage is derived in

$$
\mathrm{PDL}_{V, \mathrm{OPT}}=\frac{P_{V, T} r_{L}}{r_{R}^{\prime}+\left(1 / F_{2}\right)} .
$$

The $i$ th currents of $\mathbf{I}_{V, I i}$ and $\mathbf{I}_{V, I i, \mathrm{OPT}}$ flowing into $\mathrm{TX}_{i}$ for $\mathrm{PDL}_{V, I}$ and $\mathrm{PDL}_{V, \text { OPT }}$ can be derived by substituting $\mathbf{V}_{V, S, I}$ and $\mathbf{V}_{V, S i \text { OPT }}$ into equations (16a), (16b), and (16c), respectively.

$$
\begin{gathered}
\mathbf{I}_{V, I i}=\left(\omega_{0} C_{C i}\right)^{2} r_{T i} \mathbf{V}_{V, S, I}+\omega_{0}^{2} C_{C i} r_{R}^{\prime} Q_{T i R} \sum_{j=1}^{n} C_{C j} Q_{T j R} \mathbf{V}_{V, S, I}+j \omega_{0}^{3} C_{C i} \sum_{j=1, j \neq i}^{n} C_{C j} M_{T i T j} \mathbf{V}_{V, S, I}, \\
\mathbf{I}_{V, I i, \mathrm{OPT}}=\left(\omega_{0} C_{C i}\right)^{2} r_{T i} \mathbf{V}_{V, S i, \mathrm{OPT}}+\omega_{0}^{2} C_{C i} r_{R}^{\prime} Q_{T i R} \sum_{j=1}^{n} C_{C j} Q_{T j R} \mathbf{V}_{V, S j, \mathrm{OPT}}+j \omega_{0}^{3} C_{C i} \sum_{j=1, j \neq i}^{n} C_{C j} M_{T i T j} \mathbf{V}_{V, S j, \mathrm{OPT}} .
\end{gathered}
$$

\section{Analysis and Comparison of the Two Optimization Schemes}

4.1. Clear Relationships between the Two Optimization Schemes. The presented analysis in Sections 2 and 3 has shown some differences between current- and voltage-optimized schemes. The parameters of $\mathrm{PDL}_{C}, P_{C, T}, \mathbf{I}_{C, T, I}, \mathrm{PDL}_{C, I}, \mathbf{I}_{C, T i, \mathrm{OPT}}$, and $\mathrm{PDL}_{C, \mathrm{OPT}}$ for the current-optimized scheme correspond to $\mathrm{PDL}_{V}, P_{V, T}, \mathbf{V}_{V, S, I}, \mathrm{PDL}_{V, I}, \mathbf{V}_{V, S i, \mathrm{OPT}}$, and $\mathrm{PDL}_{V, \mathrm{OPT}}$ for the voltage-optimized scheme. The optimization goals are currents in TXs for the current-optimized scheme, while the counterparts are voltages supplied by sources for the voltage-optimized scheme. The parameters of $\mathrm{PDL}_{C, I}\left(\mathrm{PDL}_{C, \mathrm{OPT}}\right)$ and $\mathrm{PDL}_{V, I}$ $\left(\mathrm{PDL}_{V, \mathrm{OPT}}\right)$ have the same expressions, namely, $\mathrm{PDL}_{I}=\mathrm{PDL}_{C, I}=\mathrm{PDL}_{V, I} \quad$ and $\quad \mathrm{PDL}_{\mathrm{OPT}}=\mathrm{PDL}_{I, \mathrm{OPT}}=$ $\mathrm{PDL}_{V, \mathrm{OPT}}$; if $P_{C, T}=P_{V, T}=P_{T}$, we obtain

$$
\begin{gathered}
\mathrm{PDL}_{I}=\mathrm{PDL}_{C, I}=\mathrm{PDL}_{V, I}=\frac{P_{T} r_{L}}{r_{R}^{\prime}+\left(1 / F_{1}\right)}, \\
\mathrm{PDL}_{\mathrm{OPT}}=\mathrm{PDL}_{I, \mathrm{OPT}}=\mathrm{PDL}_{V, \mathrm{OPT}}=\frac{P_{T} r_{L}}{r_{R}^{\prime}+\left(1 / F_{2}\right)} .
\end{gathered}
$$

Compared with $I_{C, T, I}\left(I_{C, T i, \mathrm{OPT}}\right)$ in the current-optimized scheme, the expression form of $V_{V, S, I}\left(V_{V, S i, \mathrm{OPT}}\right)$ in the voltage-optimized scheme adds parameters of resonant frequency, $\omega_{0}$, and shunt compensation capacitance, $C_{C i}$. When the TXs are the same, namely, that $r_{T i}=r_{T}$ and $C_{C i}=$ $C_{C}$ are true, we obtain the relations between $I_{C, T, I}\left(I_{C, T i, \mathrm{OPT}}\right)$ and $V_{V, S, I}\left(V_{V, S i, \mathrm{OPT}}\right)$ under $P_{C, T}=P_{V, T}=P_{T}$ :

$$
\begin{aligned}
\mathbf{V}_{V, S, I} & =\frac{\mathbf{I}_{C, T, I}}{\omega_{0} C_{C}}=\frac{\sqrt{P_{T}}}{\omega_{0} C_{C} \sqrt{n r_{T}+r_{R}^{\prime}}\left(\sum_{i=1}^{n} Q_{T i R}\right)^{2}}, \\
\mathbf{V}_{V, S i, \mathrm{OPT}} & =\frac{\mathbf{I}_{C, T i, \mathrm{OPT}}}{\omega_{0} C_{C}}=\frac{\sqrt{P_{T}} Q_{T i R}}{\omega_{0} C_{C} \sqrt{\left(\sum_{i=1}^{n} Q_{T i R}^{2}\right)\left(r_{T}+r_{R}^{\prime} \sum_{i=1}^{n} Q_{T i R}^{2}\right)}} .
\end{aligned}
$$

Under the above conditions of $r_{T i}=r_{T}, C_{C i}=C_{C}$, and $P_{C, T}=P_{V, T}=P_{T}$, equations (24a) and (24b) can be rewritten by combining them with (26a) and (26b) as

$$
\begin{gathered}
\mathbf{I}_{V, I i}=\omega_{0} C_{C} \mathbf{V}_{C, S i}, \\
\mathbf{I}_{V, I i, \mathrm{OPT}}=\omega_{0} C_{C} \mathbf{V}_{C, S i, \mathrm{OPT}} .
\end{gathered}
$$

4.2. Discussion. The clear relationships between the current- and voltage-optimized scheme are presented in equations (26a), (26b) and (27a), (27b). For a given $P_{T}$, $\mathbf{I}_{C, T, I}$ and $\mathbf{I}_{C, T i, \mathrm{OPT}}$ for $\mathrm{PDL}_{I}$ and $\mathrm{PDL}_{\mathrm{OPT}}$, respectively, are presented in Figure 3. The corresponding $\mathrm{PDL}_{I}$ and $\mathrm{PDL}_{\mathrm{OPT}}$ versus $D_{M}$ are also shown in Figure 4. According to the two optimal methods proposed in Sections 2 and 3, $\mathrm{PDL}_{\mathrm{OPT}}$ for the two optimal cases significantly outperformed $\mathrm{PDL}_{I}$ for EC and EV cases. Therefore, we focus on the two optimal cases to present the differences between them in the next discussions.

It can be found from the relationships between $\mathbf{V}_{V, S i \text { OPT }}$ in OV Case and $\mathbf{I}_{C, T i, \text { OPT }}$ in OE Case in equation (26b) that the optimal $\mathbf{V}_{V, S i \text {,OPT }}$ can be adjusted by changing the shunt capacitance values of $C_{C}$. It is for the same reason that $\mathbf{I}_{V, I i \text {,OPT }}$ in OV Case can be adjusted by adding different $C_{C}$. Therefore, the OV Case using LCC compensation networks for the multiple-TX system can not only feed the same PDL as OC Case but also can adjust the feeding voltages and currents according to the condition that the maximum voltage or current endurance for circuit element is limited. As shown in Figure 6, we present the source voltages of $\mathbf{V}_{C, S i, \text { OPT }}$ and $\mathbf{V}_{V, S i, O P T}$ and feeding currents of $\mathbf{I}_{C, T i \text {,OPT }}$ and $\mathbf{I}_{V, I i, \mathrm{OPT}}$ versus $D_{M}$ for different shunt capacitance values of $C_{C}$ when $D_{T}=0.3 \mathrm{~m}$ and $P_{T}=20 \mathrm{~W}$. As can be seen from the illustration, the source voltages and feeding currents of $\mathbf{V}_{C, S i, \mathrm{OPT}}$ and $\mathbf{I}_{C, T i, \mathrm{OPT}}$ in OC Case are shown using the black curve with no changes when the system structure and load impedance are predetermined, while $\mathbf{V}_{V, S i, \mathrm{OPT}}$ and $\mathbf{I}_{V, I i, \mathrm{OPT}}$ in OV Case can be controlled via regulating the values of shunt capacitance $C_{C}$ to maintain the expected power output as shown in the annotation of 5-TX System and $D_{T}=0.3 \mathrm{~m}$ in Figure $4(\mathrm{~b}) . \mathbf{V}_{V, S i, \mathrm{OPT}}$ decreases and $\mathbf{I}_{V, I i, \text { OPT }}$ increases with increasing $C_{C}$ to ensure that $P_{T}=20 \mathrm{~W}$ is true. 


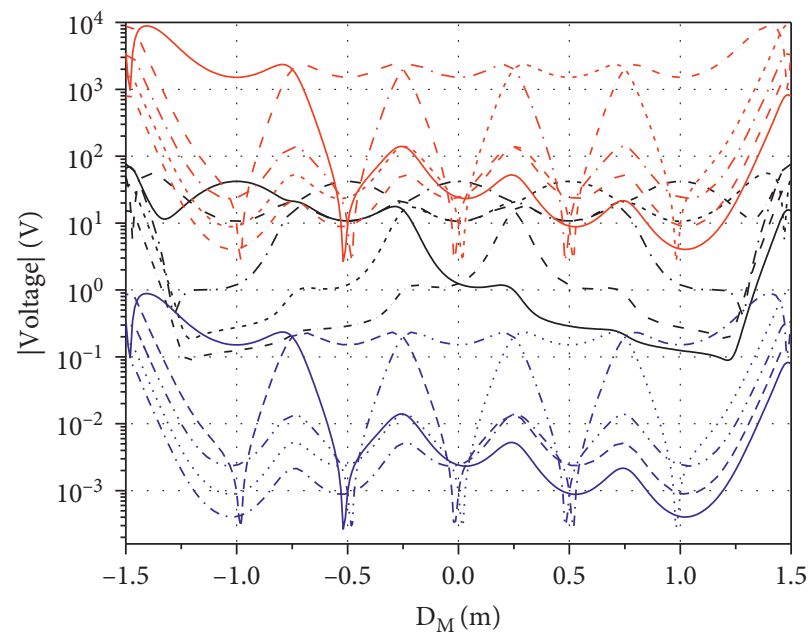

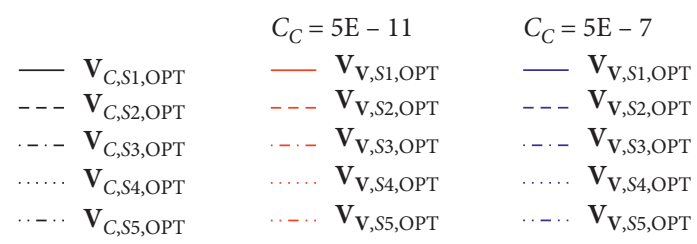

(a)

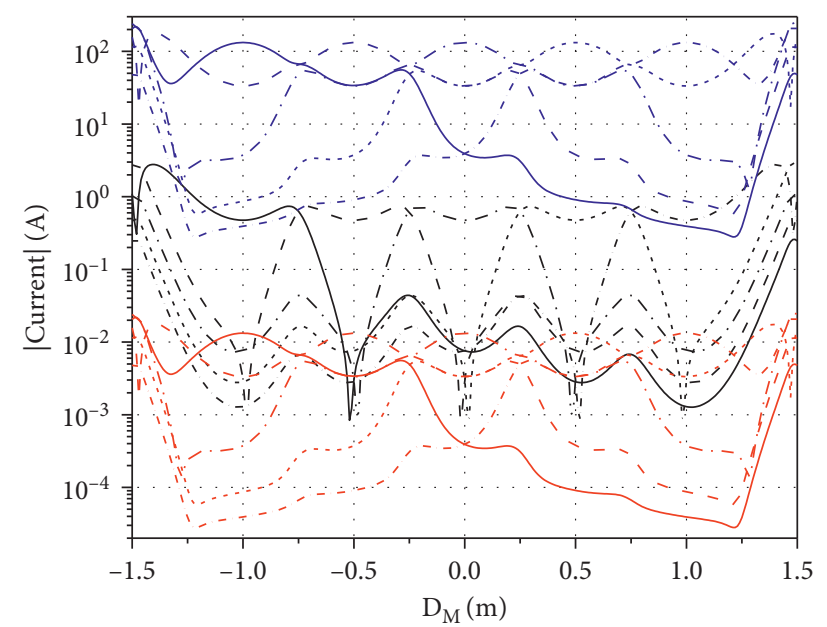

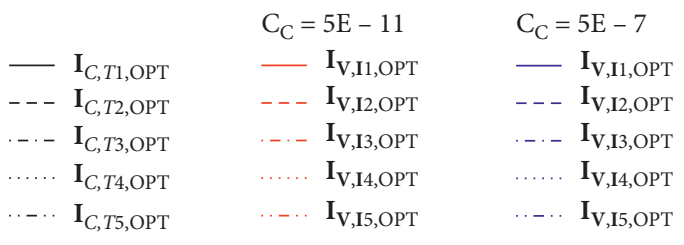

(b)

FIgURe 6: Comparison of source voltage and feeding current between OC and OE Case for 5-TX system when $D_{T}=0.3 \mathrm{~m}$ and $P_{T}=20 \mathrm{~W}$. (a) Optimal voltage and (b) current for maximizing PDL.

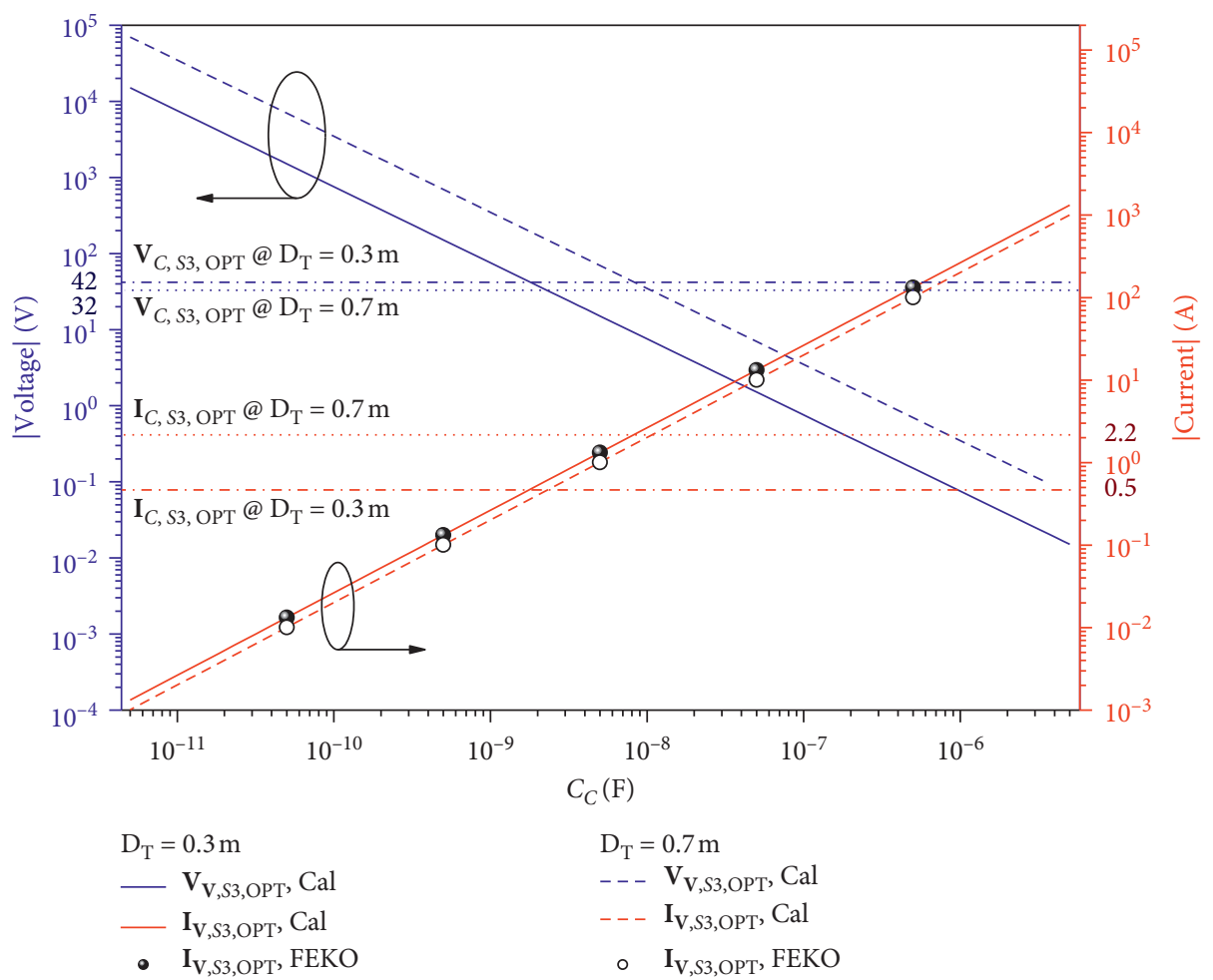

FIGURE 7: Simulated and calculated source voltage and feeding current in $\mathrm{TX}_{3}$ versus shunt capacitance for maximizing PDL in OV Case. 


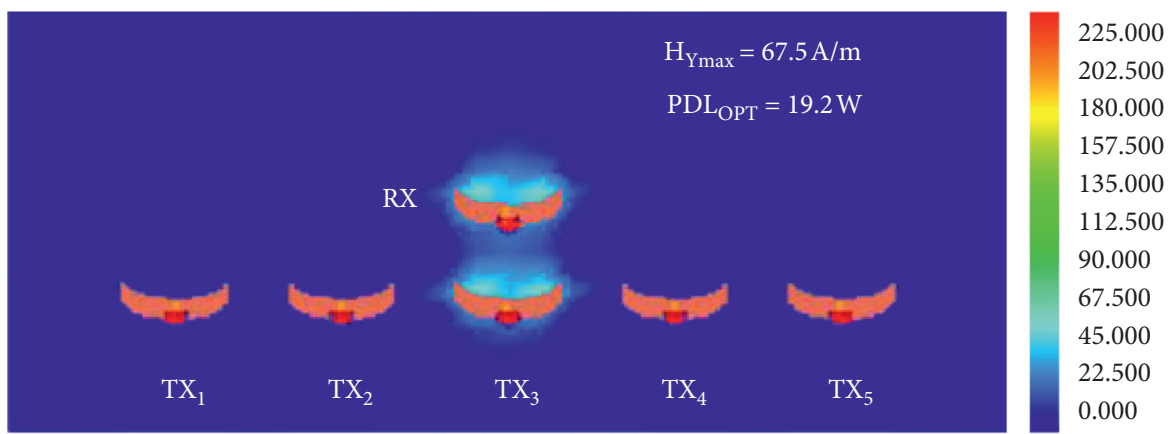

(a)

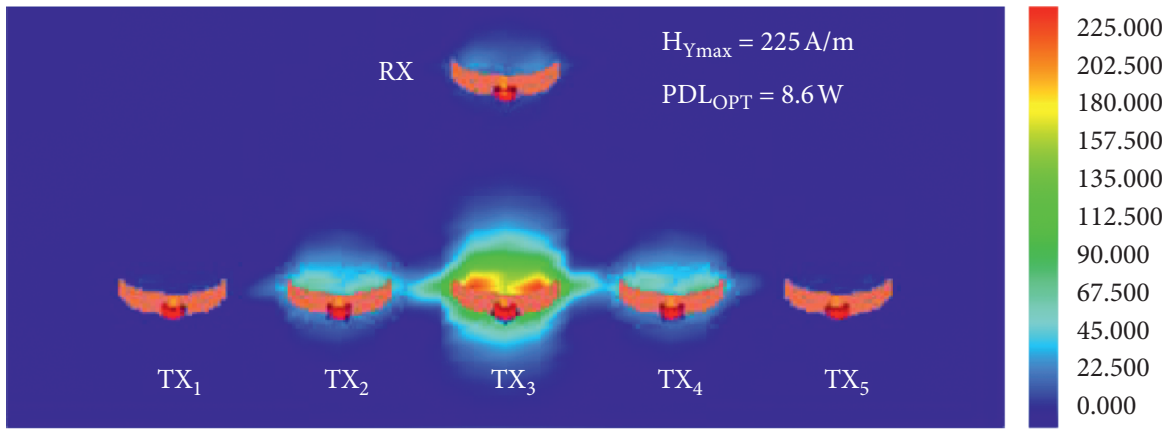

(b)

Figure 8: The distribution of magnetic fields of 5-TX WPT system for OV or OC Case. (a) $D_{T}=0.3$ (m). (b) $D_{T}=0.7 \mathrm{~m}$.

\section{Theoretical Calculation and Full-Wave Electromagnetic Simulation Verification}

To verify the effectiveness of the optimal method for maximizing the PDL and the technology of adjusting current and voltage in the multiple-TX WPT system, a WPT system with five identical TXs and a single RX is modeled in this section. The electrical parameters of the enumerated WPT system here are the same as those used in Section 2.2. Figure 7 shows the simulated and calculated source voltage and feeding current in $\mathrm{TX}_{3}$ for maximizing PDL under input power $P_{T}=20 \mathrm{~W}$. The solid and dashed lines represent the optimal voltage and current at $D_{T}=0.3$ and $0.7 \mathrm{~m}$, respectively. The values of source voltage at $D_{T}=0.7 \mathrm{~m}$ are always higher than those at $D_{T}=0.3 \mathrm{~m}$. But correspondingly, the values of feeding current at the former distance are always lower than those at the latter distance. This is because the input and output power always hold constant in the transient process of the shunt capacitance $C_{C}$. For FEKO simulation, the calculated values of source voltage are used and input impedance matching is implemented in the WPT model. The simulated values are shown in the form of scattered dots, which are accorded with the theoretical calculating values. The source voltage and feeding current are fixed at $42 \mathrm{~V}$ and $0.5 \mathrm{~A}$ for OC Case at $D_{T}=0.3 \mathrm{~m}$, and the counterparts are fixed at $32 \mathrm{~V}$ and $2.2 \mathrm{~A}$ at $D_{T}=0.7 \mathrm{~m}$. From the above, we can see that the OV Case can effectively regulate the source voltage and feeding current by comparing the OC Case.

Setting the source voltage based on equation (14b) in the OC Case and equation (22) in OV Case, we obtain the same current flowing in TXs for both cases. Figure 8 shows the magnetic flux densities in a setting plane which is perpendicular to the planes of coils. $\mathrm{PDL}_{\mathrm{OPT}}=19.2 \mathrm{~W}$ at $D_{T}=0.3 \mathrm{~m}$ is larger than $\mathrm{PDL}_{\mathrm{OPT}}=8.6 \mathrm{~W}$ at $D_{T}=0.7 \mathrm{~m}$. However, the maximum axial magnetic field $H_{Y \max }=67.5 \mathrm{~A} /$ $\mathrm{m}$ at the former position is lower than $H_{Y \max }=225 \mathrm{~A} / \mathrm{m}$ at the latter position. This is due to the fact that the maximum magnetic flux density appears around $\mathrm{TX}_{3}$ at $D_{T}=0.7 \mathrm{~m}$, which is confirmed by the maximum feeding current flowing in $\mathrm{TX}_{3}$ at $D_{T}=0.7 \mathrm{~m}$ case. The feeding currents are investigated in Figures $3(\mathrm{c})$ and $3(\mathrm{~d})$ with the legend of 5-TX System.

\section{Conclusions}

The multiple-TX and single-RX WPT system is optimized based on current- and voltage-optimized circuit scheme in this paper. In the section of the current-optimized circuit scheme, we present the equal current case for comparing to illustrate the advantages of the proposed optimal current solution. In order to use voltage source with finite output voltage or avoid overcurrent to damage circuit element, the adjustable method for the source voltage and feeding current is proposed based on voltage-optimized circuit scheme. The analysis and relations between the two optimization schemes are presented in later parts of the paper. Based on the electromagnetic simulation software FEKO, the three-dimensional full-wave simulation is implemented to verify the two optimization solutions.

\section{Data Availability}

The data used to support the findings of this study are included within the article. 


\section{Conflicts of Interest}

The authors declare that they have no conflicts of interest.

\section{Acknowledgments}

Jin Zhang would like to thank Dr. Dong Chen for helpful discussions for improving this paper. This research was supported in part by the Natural Science Foundation of the Jiangsu Higher Education Institutions of China(Grant no. 19KJB510029), the open research fund of the National and Local Joint Engineering Laboratory of RF Integration and Micro-Assembly Technology (KFJJ20180203), the Scientific Research Foundation for the High-Level Talents of Jinling Institute of Technology (jit-b-201719), and the Scientific Research Incubation Foundation of Jinling Institute of Technology (jit-fhxm-201802).

\section{References}

[1] K. André, K. Aristeidis, M. Robert, J. D. Joannopoulos et al., "Wireless power transfer via strongly coupled magnetic resonances," Science, vol. 317, pp. 83-86, 2007.

[2] A. P. Sample, D. A. Meyer, and J. R. Smith, "Analysis, experimental results, and range adaptation of magnetically coupled resonators for wireless power transfer," IEEE Transactions on Industrial Electronics, vol. 58, no. 2, pp. 544-554, 2011.

[3] J. Zhang and C. Cheng, "Quantitative investigation into the use of resonant magneto-inductive links for efficient wireless power transfer," IET Microwaves, Antennas \& Propagation, vol. 10, no. 1, pp. 38-44, 2016.

[4] F. Zhang, S. A. Hackworth, W. Fu, C. Li, Z. Mao, and M. Sun, "Relay effect of wireless power transfer using strongly coupled magnetic resonances," IEEE Transactions on Magnetics, vol. 47, no. 5, pp. 1478-1481, 2011.

[5] D. Ahn and S. Hong, "A study on magnetic field repeater in wireless power transfer," IEEE Transactions on Industrial Electronics, vol. 60, no. 1, pp. 360-371, 2013.

[6] W. Zhong, C. K. Lee, and S. Y. R. Hui, "General analysis on the use of Tesla's resonators in domino forms for wireless power transfer," IEEE Transactions on Industrial Electronics, vol. 60, no. 1, pp. 261-270, 2013.

[7] J. Zhang and C. Cheng, "Analysis and optimization of threeresonator wireless power transfer system for predeterminedgoals wireless power transmission," Energies, vol. 9, no. 4, p. 274, 2016.

[8] M. Fu, H. Yin, M. Liu, Y. Wang, and C. Ma, “A 6.78 MHz multiple-receiver wireless power transfer system with constant output voltage and optimum efficiency," IEEE Transactions on Power Electronics, vol. 33, no. 6, pp. 5330-5340, 2018.

[9] P. Hao, L. Lu, and Z. Liang, "Priority evaluation for multiple receivers in wireless power transfer based on magnetic resonance," in Proceedings of the IEEE Wireless Power Transfer Conference (WPTC), pp. 1-4, IEEE, Aveiro, Portugal, May 2016.

[10] U. Pratik, B. J. Varghese, A. Azad et al., "Optimum design of decoupled concentric coils for operation in double-receiver wireless power transfer systems," IEEE Journal of Emerging and Selected Topics in Power Electronics, vol. 7, no. 3, pp. 1982-1998, 2018.
[11] J. Zhang and F. Wang, "Efficiency analysis of multipletransmitter wireless power transfer systems," International Journal of Antennas and Propagation, vol. 2018, Article ID 3415239, 11 pages, 2018.

[12] C. Zhang, D. Lin, and S. Y. Hui, "Basic control principles of omnidirectional wireless power transfer," IEEE Transactions on Power Electronics, vol. 31, pp. 5215-5227, 2016.

[13] J. Zhang, C. Cheng, K. Chen, F. Wang, and Y. Gao, "Analysis of multiple-TX WPT systems using KVL and RX-side RLT," IET Microwaves, Antennas \& Propagation, vol. 13, no. 12, pp. 1997-2004, 2019.

[14] J. Zhang, D. Chen, and C. Zhang, "Enhanced power transmission for on-road AGV wireless charging systems using a current-optimized technique," Progress In Electromagnetics Research C, vol. 96, pp. 205-214, 2019.

[15] M. Kiani and M. Ghovanloo, "The circuit theory behind coupled-mode magnetic resonance-based wireless power transmission," IEEE Transactions on Circuits and Systems I: Regular Papers, vol. 59, no. 9, pp. 2065-2074, 2012.

[16] J. Jadidian and D. Katabi, "Magnetic MIMO: how to charge your phone in your pocket," in Proceedings of the International Conference on Mobile Computing and Networking, pp. 495-506, Los Angeles, CA, USA, Sepember 2014.

[17] M. R. V. Moghadam and R. Zhang, "Node placement and distributed magnetic beamforming optimization for wireless power transfer," IEEE Transactions on Signal and Information Processing Over Networks, vol. 4, no. 2, pp. 264-279, 2018.

[18] G. Yang, M. R. V. Moghadam, and R. Zhang, "Magnetic beamforming for wireless power transfer," in Proceedings of the IEEE International Conference on Acoustics, Speech and Signal Processing (ICASSP), pp. 3936-3940, IEEE, Shanghai, China, March 2016.

[19] J. Lee and S. Nam, "Fundamental aspects of near-field coupling small antennas for wireless power transfer," IEEE Transactions on Antennas and Propagation, vol. 58, pp. 3442-3449, 2010.

[20] J. Zhang and C. Cheng, "Investigation of near-field wireless power transfer between two efficient electrically small planar antennas," in Proceedings of the IEEE 3rd Asia-Pacific Conference on Antennas and Propagation (APCAP), pp. 720-723, Harbin, China, July 2014.

[21] Z. Chen, H. Sun, and W. Geyi, "Maximum wireless power transfer to the implantable device in the radiative near field," IEEE Antennas and Wireless Propagation Letters, vol. 16, pp. 1780-1783, 2017.

[22] X. Dai, J. C. Jiang, and J. Q. Wu, "Charging area determining and power enhancement method for multiexcitation unit configuration of wirelessly dynamic charging EV system," IEEE Transactions on Industrial Electronics, vol. 66, no. 5, pp. 4086-4096, 2018.

[23] Q. T. Duong and M. Okada, "kQ-product formula for multiple-transmitter inductive power transfer system," IEICE Electronics Express, vol. 14, pp. 1-8, 2017.

[24] A. K. RamRakhyani, S. Mirabbasi, and M. Chiao, "Design and optimization of resonance-based efficient wireless power delivery systems for biomedical implants," IEEE Transactions on Biomedical Circuits and Systems, vol. 5, no. 1, pp. 48-63, 2010.

[25] S. Zhou and C. C. Mi, "Multi-paralleled LCC reactive power compensation networks and their tuning method for electric vehicle dynamic wireless charging," IEEE Transactions on Industrial Electronics, vol. 63, no. 10, pp. 6546-6556, 2015.

[26] W. Kim and D. Ahn, "Efficient deactivation of unused LCC inverter for multiple transmitter wireless power transfer," IET Power Electronics, vol. 12, no. 1, pp. 72-82, 2018. 